\title{
Preventable harm: getting the measure right
}

\author{
Patient perspectives are essential to reliable detection of harm, including near misses
}

\author{
Irene Papanicolas associate professor ${ }^{1}$, Jose F Figueroa assistant professor ${ }^{2}$ \\ ${ }^{1}$ Department of Health Policy, London School of Economics and Political Science, London WC2A 2AE, UK; ${ }^{2}$ Department of Medicine, Harvard \\ Medical School, Brigham and Women's Hospital, Boston, MA, USA; Correspondence to: I Papanicolas I.N.Papanicolas@Ise.ac.uk
}

Healthcare is not as safe as it should be. Twenty years since the publication of the seminal report To Err is Human, ${ }^{1}$ Panagioti and colleagues (doi:10.1136/bmj.14185) estimate that about $12 \%$ of patients still experience some form of harm associated with healthcare, around half of which is preventable. ${ }^{2}$ This study raises serious concerns about the safety of health systems. How should health system leaders, doctors, and patients interpret these findings?

\section{What is preventable harm?}

Separating harm into outcomes that are deemed either preventable or inevitable is a necessary step to advance efforts toward patient safety. Yet no consensus exists as to what constitutes preventable harm, and even experienced clinicians vary in the extent to which they agree on whether an error is preventable. ${ }^{3}$ Panagioti and colleagues define preventable harm as the result of an identifiable modifiable cause and an event the recurrence of which can be avoided by the adaptation of a process or adherence to guidelines. This approach, although reasonable, has important limitations.

This measure relies on evidence linking a particular process of care or a particular standard of care to the avoidance of harm. Patient harm, however, is usually caused by the interplay of many linked failings across the system, not just a missed process of care. Root cause analyses show that most instances of patient harm result from a combination of individual failings, systemic weaknesses, and environmental factors. ${ }^{4}$ A model focused on defining preventability by linking an identifiable process to harm would be less likely to capture events that result from multiple failings-including, for example, poor teamwork or communication-and perhaps overemphasise harm resulting from individual failings.

In addition, many complications are only partially preventable, and therefore distinguishing the preventable from the inevitable becomes difficult. Even after implementation of system-wide prophylaxis against venous thromboembolism (VTE), for example, rates of VTE events, which are considered potentially preventable, might not improve. ${ }^{5}$ Finally, evidence and care are constantly changing. What is considered inevitable harm today could in fact be preventable in five years. Definitions of preventable harm need to be continually updated.

\section{Better measurement}

Firstly, it is important to capture all potential risks to patient safety, not just adverse events. Not all lapses in safety result in harm but instead are "near misses." It is likely that the root causes of near misses and adverse events are similar. ${ }^{6}$ Near misses are, however, often underreported—an important missed opportunity for health systems and providers to better understand how safety can be improved. We need a culture that strongly encourages the diligent reporting of near misses, all of which are learning opportunities for staff and systems.

A second key strategy is to improve the ability to detect harm across all settings. Despite concerns around the prevalence of patient harm across health systems globally, the measurement of harm is often concentrated among a few high income countries and from limited care settings. In the systematic review by Panagioti and colleagues, $86 \%$ of the studies were from America or Europe and 78\% reported estimates from the hospital or emergency room setting. More systematic measurement of harm in other settings is essential, including primary care, where common causes of harm, such as diagnostic errors, are more likely but are more difficult to measure.

A third strategy is to increase patient and public engagement in identifying causes of preventable harm. Most studies included in the systematic review were retrospective assessments of medical charts, whereas few drew on information from patient reports. Yet the patient's perspective is essential. Patients and their families can capture important information from their experience of care, which can be crucial to understanding factors that led to harm, such as lapses in communication, staffing issues, and the care environment. ${ }^{3}$ Exploring the further use of patient reported measures might improve detection of different forms of harm and provide a new means of measurement in settings with a more limited data infrastructure.

The study by Panagioti and colleagues serves as a reminder of the extent to which medical harm is prevalent across health systems, and, importantly, draws attention to how much is potentially preventable. Moving forward, efforts need to be focused on improving the ability to measure preventable harm. This includes fostering a culture that allows for more systematic capturing of near misses, identifying harm across multiple care settings and countries, and empowering patients to help ensure a safe and effective health system. 
Competing interests: We have read and understood the BMJ Group policy on declaration of interests and declare the following interests: none.

Provenance and peer review: Commissioned; not peer reviewed.

1 Kohn LT, Corrigan JM, Donaldson MS, eds. To err is human: building a safer health system. Institute of Medicine, National Academy Press, 1999.

2 Panagioti M, Khan K, Keers RN, etal . Prevalence, severity, and nature of preventable patient harm across medical care settings: systematic review and meta-analysis. $B M J$ 2019;366:14185.

3 O'Hara JK, Reynolds C, Moore S, etal . What can patients tell us about the quality and safety of hospital care? Findings from a UK multicentre survey study. BMJ Qual Saf 2018:27:673-82. 10.1136/bmjqs-2017-006974. 29545325
4 Corwin GS, Mills PD, Shanawani H, Hemphill RR. Root Cause Analysis of ICU Adverse Events in the Veterans Health Administration. Jt Comm J Qual Patient Saf 2017;43:580-90. 10.1016/j.jcjq.2017.04.009. 29056178

5 Heit JA, Crusan DJ, Ashrani AA, Petterson TM, Bailey KR. Effect of a near-universal hospitalization-based prophylaxis regimen on annual number of venous thromboembolism events in the US. Blood 2017;130:109-14. 10.1182/blood-2016-12-758995. 28483763 6 Sheikhtaheri A. Near Misses and Their Importance for Improving Patient Safety. Iran J Public Health 2014;43:853-4.26110160

Published by the BMJ Publishing Group Limited. For permission to use (where not already granted under a licence) please go to http://group.bmj.com/group/rights-licensing/ permissions 